\section{Fatal metabolic decompensation in carbonic anhydrase VA deficiency despite early treatment and control of hyperammonemia}

To the Editor:

Carbonic anhydrase VA deficiency (OMIM 615751) is a rare autosomal recessive inborn error of metabolism that leads to acute metabolic crises in infancy and early childhood. Biallelic variants in CA5A have been identified as the underlying genetic defect. So far, only 14 patients have been reported indicating that the actual phenotypic spectrum is not represented in the literature to date. ${ }^{1,2}$ Ten of these were reported by Diez-Fernandez et al. in Genetics in Medicine. ${ }^{2}$ Disease features include encephalopathy as well as hyperammonemia, lactic acidosis, ketonuria, and hypoglycemia. In about two-thirds of the patients reported so far, disease manifestation was limited to a single metabolic decompensation, after which the patients remained metabolically stable. ${ }^{2}$ The other third of patients exhibited recurrent metabolic crises triggered by catabolism. Treatment measures of these metabolic decompensations have been suggested to include generation of anabolism via glucose and lipid administration as well as lowering ammonia levels using agents such as sodium benzoate. Anecdotal evidence suggests an additional positive effect of carglumic acid with regard to lowering ammonia levels more rapidly. All patients published so far survived without severe neurological sequelae and the general prognosis after initial metabolic decompensation seemed to be good.

An 18-month-old patient was admitted to our intensive care unit. He had been born preterm at 26 weeks but had an otherwise unremarkable medical record and, thus far, normal psychomotor development. He presented with acute lethargy, vomiting, and seizures. Laboratory analyses revealed hyperammonemia $(460 \mu \mathrm{mol} / \mathrm{L}$; reference range $6-50)$ as well as metabolic acidosis $\left(\mathrm{pH} 7,3\right.$; base excess $-17 \mathrm{mmol} / \mathrm{L}, \mathrm{pCO}_{2}$ $15 \mathrm{mmHg})$ with elevated lactate levels $(10 \mathrm{mmol} / \mathrm{L}$; reference range $0.5-1)$. Meningitis was excluded and an initial cranial magnetic resonance image (MRI) revealed no remarkable alterations. Additionally, the patient displayed arterial hypertension. Protein and fat intake were immediately stopped and the patient received intravenous glucose. Furthermore, the patient was treated with drugs to lower ammonia levels. These included sodium benzoate, sodium phenylacetate, L-arginine, as well as carglumic acid. Cranial MRI was repeated 12 hours after the initial investigation and did not reveal novel alterations. A lactate peak was detected in basal ganglia in magnetic resonance spectroscopy. Hyperammonemia and metabolic acidosis resolved within a little less than 24 hours. However, encephalopathy and arterial hypertension persisted and worsened even after normalization of metabolic parameters. The patient eventually required mechanical ventilation due to respiratory failure with insufficient oxygenation. On the third day of admission the patient displayed repetitive seizures despite continued anticonvulsive medication with levetiracetam, severe hypernatremia $(>150 \mathrm{mmol} / \mathrm{L})$, and his pupils were widened and ceased to react to light. An additional cranial MRI revealed severe brain herniation. After the MRI, a probe was surgically inserted to measure intracranial pressure, which was measured above $80 \mathrm{mmHg}$. Under medical treatment for cranial hypertension, intracranial pressure further increased and the patient eventually passed away. Exome sequencing revealed a homozygous deletion of the first two exons of the CA5A gene. No other genetic variants could be identified that would explain the rapid disease course.

Apart from adding to the list of possible variants in carbonic anhydrase deficiency, this case demonstrates that the disease course can be fulminant and fatal despite rapid initiation of adequate treatment and despite quick normalization of ammonia and lactate levels during metabolic crisis. So far, the prognosis of this disease has been considered quite good. We suggest this view should be handled with caution when counseling the parents. Furthermore, it is remarkable that the patient remained asymptomatic throughout the course of treatment for premature birth during the first months of life. This suggests the possibility of a compensatory mechanism to avoid metabolic decompensation, which seems to fail at some point due to an unknown internal or external trigger.

\section{DISCLOSURE}

The authors declare no conflicts of interest.

Publisher's note Springer Nature remains neutral with regard to jurisdictional claims in published maps and institutional affiliations.

\section{Fabian Baertling, $M D^{1}$, Matias Wagner, $M D$ (iD $^{2,3,4}$, Theresa Brunet ${ }^{2}$, Hemmen Sabir, $M D^{1}$, Dagmar Wieczorek, $M D^{5}$, Thomas Meitinger, $M D^{2,3}$, Thomas Meissner, $M D^{1}$ and Felix Distelmaier, $M D^{1}$}

${ }^{1}$ Department of General Pediatrics, Neonatology and Pediatric Cardiology, HeinrichHeine-University, Düsseldorf, Germany; ${ }^{2}$ Institute of Human Genetics, Technical University München, Munich, Germany; ${ }^{3}$ Institute of Human Genetics, Helmholtz Zentrum München, Neuherberg, Germany; ${ }^{4}$ Institute of Neurogenomics, Helmholtz Zentrum München, Neuherberg, Germany; ${ }^{5}$ Institute of Human Genetics, Medical 
Faculty, Heinrich Heine University, Düsseldorf, Germany. Correspondence: Felix Distelmaier (felix.distelmaier@med.uni-duesseldorf.de)

\section{REFERENCES}

1. Van Karnebeek CD, Sly WS, Ross CJ, et al. Mitochondrial carbonic anhydrase VA deficiency resulting from CA5A alterations presents with hyperammonemia in early childhood. Am J Hum Genet. 2014;94: 453-61.
2. Diez-Fernandez $C$, Rüfenacht $V$, Santra $S$, et al. Defective hepatic bicarbonate production due to carbonic anhydrase VA deficiency leads to early-onset life-threatening metabolic crisis. Genet Med. 2016;18: 999-1000.

Advance online publication 22 October 2019. doi:10.1038/s41436-019-0677-9 